\title{
Métodos numéricos aplicados a ensayos de terreno para la determinación de la permeabilidad saturada
}

\section{Numerical methods applied to field tests for the determination of the saturated permeability}

Fecha de entrega: 28 de noviembre 2014 Fecha de aceptación: 15 de mayo 2015

\section{Julio Tejo ${ }^{1}$, Marcelo González ${ }^{2}$ y Adam Darby ${ }^{1}$ \\ ${ }^{1}$ Golder Associates S.A, Magdalena 181, piso 3, Las Condes, Chile, jtejo@golder.cl, adarby@golder.cl \\ ${ }^{2}$ CODELCO S.A, Av. Libertador Bernardo O’Higgins 1449 SDT2, piso 8, Santiago, Chile, mgonz108@codelco.cl}

Como parte de un estudio de infiltración de un nuevo depósito de relaves en el norte de Chile emplazado sobre depósitos aluviales consolidados y no saturados, se ha llevado a cabo una caracterización hidrogeológica basada en una campaña de investigación de terreno. Se contempló la ejecución de sondajes con ensayos de permeabilidad de carga constante y carga variable; ensayos de laboratorio para caracterizar los materiales encontrados; y trabajos de gabinete enfocados en el desarrollo de análisis numéricos. Se desarrollaron diversos modelos numéricos que en conjunto con las propiedades de los materiales ensayados de los sondajes, permitieron estimar los valores de permeabilidad saturada del subsuelo, valores que pueden ser subsecuentemente incorporados en los modelos hidrogeológicos del depósito de relaves. Se realizaron modelos de infiltración para simular cada tramo de ensayo en los sondajes. Se aplicó un proceso iterativo, definiendo una curva de conductividad hidráulica versus succión, obteniendo en cada modelo, un valor de caudal igual o similar, al medido en los ensayos de terreno para las condiciones de carga constante y carga variable. La aplicación de estos métodos también se ha utilizado para optimizar los ensayos en terreno, tanto en tipología de ensayo de permeabilidad a ejecutar como en la duración requerida de los mismos.

Palabras clave: coeficiente de permeabilidad, suelos no saturados, curvas de succión, ensayo de permeabilidad en sondaje
As part of a seepage study for a new tailings facility in the north of Chile, which is to be built upon deep unsaturated consolidated alluvial deposits, an exhaustive hydrogeological characterization was carried out based on field work. Constant and falling head permeability testing in boreholes; laboratory testwork to characterize the materials; and desktop studies aimed at developing numerical models were included. A series of numerical models, which together with the soil material properties determined from the boreholes, allowed the saturated permeability of the soil to be estimated. The results were then subsequently applied in the hydrogeological modeling of the tailings facility. Infiltration models were carried out to simulate each test interval in the boreholes. An iteration process was applied, based on a soil water characteristic curve for the material, obtaining from each model flow values equal to or similar to that measured in the field for the constant and falling head permeability tests. The use of these models also allowed the field testwork to be optimized, both relating to the type of test to undertake and also the duration of each test.

Keywords: coefficient of permeability, unsaturated soils, soil water characteristic curves, borehole permeability test

\section{Introducción}

El diseño de un nuevo tranque de relaves sobre un extenso depósito no saturado en el norte de Chile, incorporó la caracterización hidrogeológica del sitio, con el fin de evaluar las tasas de infiltración desde los relaves hacia el terreno de fundación en el corto, mediano y largo plazo. Dentro de los trabajos de caracterización hidrogeológica, se llevaron a cabo ensayos de permeabilidad en sondajes, para posteriormente usar esta información en la estimación de los perfiles de permeabilidades en profundidad del 
sitio. Todas las soluciones analíticas existentes para la estimación del coeficiente de permeabilidad saturada dentro de un medio no saturado a partir de ensayos de permeabilidad realizan ciertas suposiciones, sobre todo en los métodos de ensayo generalmente disponibles en sondajes (e.g. Hvorslev, 1949; Custodio y Llamas, 1983). Esto motivó el desarrollo de este estudio, que tiene como objetivo dar a conocer una metodología de análisis mediante modelamiento numérico usando el método de elementos finitos, simulando la ejecución de los ensayos de terreno e introduciendo variables y conceptos propios de la ingeniería geotécnica en suelos no saturados.

\section{Caracterización del sitio}

El área de estudio se encuentra en el norte de Chile, en el desierto de Atacama. El sitio se caracteriza por un clima desértico con precipitaciones anuales promedio inferiores a $15 \mathrm{~mm}$. Existe escasa a nula presencia de vegetación.

\section{Investigación geotécnica}

Como parte de la caracterización hidrogeológica del sitio, se desarrolló una campaña geotécnica que incluyó los siguientes trabajos de terreno: Perforación de sondajes geotécnicos de entre 100 y $300 \mathrm{~m}$ de profundidad, incluyendo recuperación de testigos y ensayos de permeabilidad; excavación de calicatas de hasta $5 \mathrm{~m}$ de profundidad y mapeo geológico superficial de suelo y roca. En conjunto a lo anteriormente expuesto, se realizaron ensayos de laboratorio a muestras obtenidas de las calicatas y sondajes, con el objeto de caracterizar los materiales hallados y obtener parámetros geotécnicos e hidrogeológicos necesarios para la posterior caracterización hidrogeológica del sitio.

\section{Ensayos de permeabilidad en sondajes}

Se ejecutaron ensayos hidráulicos de carga constante y carga variable en los sondajes en suelo, contemplando longitudes de cámara de 10 a $20 \mathrm{~m}$ con sistemas de packer inflables (Philip, 1985; Chapuis, 1989). Para asegurar la formación de un bulbo saturado en el suelo circundante al sondaje, se efectuaron ciclos de llenado y descenso de agua. Se evaluaron diversas combinaciones de ciclos para las pruebas, optando finalmente, tras el análisis de los ensayos iniciales, por un ensayo de carga variable prolongado de hasta 12 horas de duración al final del turno de perforación, antecedida por un ensayo de carga constante de duración aproximada de entre 15 y 30 minutos.

\section{Caracterización geotécnica}

La información procedente de la ejecución de calicatas mostró la existencia en superficie de gravas limo arenosas pobremente graduadas (GP-GM según sistema de clasificación USCS) que sobreyacen a arenas limosas con grava (SP-SM y SM) densas, con presencia de finos de baja a nula plasticidad y contenidos de humedad que varían entre $0.5 \%$ a $5 \%$. Todos los suelos en el sector tienen un origen fluvial o aluvial. Un resumen de los contenidos de gravas, arenas y finos en porcentaje, presente en muestras provenientes de seis sondajes ejecutados en suelo, se entrega en la Tabla 1, donde se aprecia predominancia de las arenas. La Figura 1 presenta su distribución en profundidad.

Tabla 1: Distribución granulométrica en muestras de sondajes

\begin{tabular}{|l|c|c|c|c|}
\hline \multirow{2}{*}{$\begin{array}{l}\text { Cantidad } \\
\text { de muestras }\end{array}$} & \multirow{2}{*}{ Material } & \multicolumn{3}{|c|}{ Contenido, \% } \\
\cline { 2 - 5 } & & Máximo & Mínimo & Promedio \\
\hline \multirow{3}{*}{107} & Gravas (de 5 a 75 mm) & 74 & 0 & 22 \\
\cline { 2 - 5 } & Arena (de 0.075 a $5 \mathrm{~mm})$ & 95 & 18 & 58 \\
\cline { 2 - 5 } & Finos $(<0.075 \mathrm{~mm})$ & 63 & 5 & 20 \\
\hline
\end{tabular}

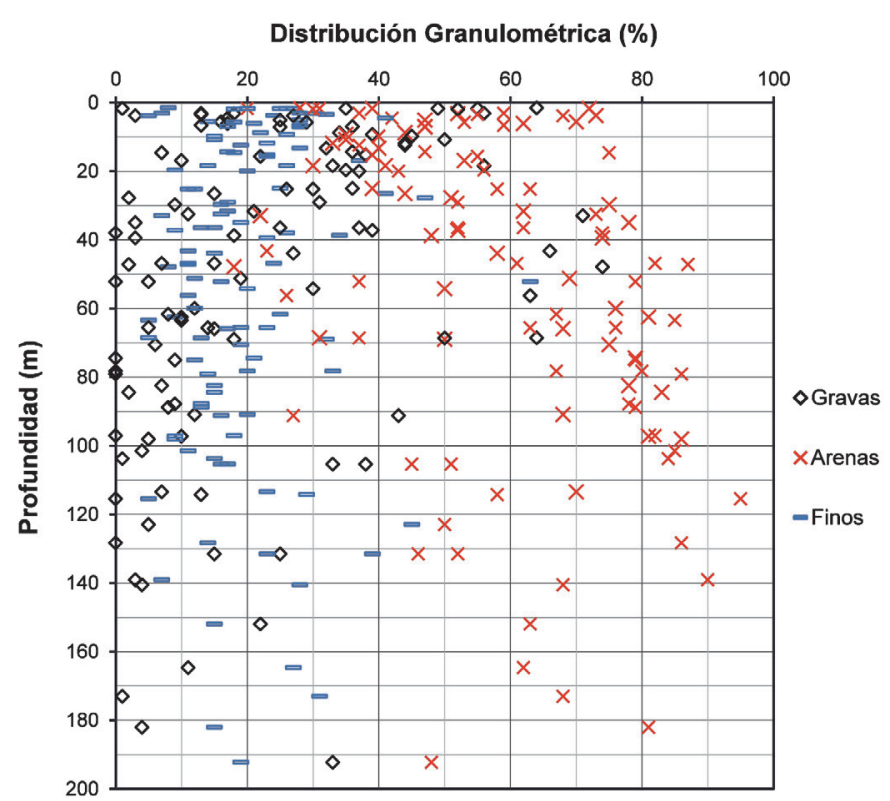

Figura 1: Distribución granulométrica en muestras de sondajes en profundidad Como se aprecia en la Figura 1, el contenido de grava disminuye a medida que aumenta la profundidad, del 
mismo modo que se incrementa la proporción de arenas. También se observó un creciente nivel de cementación de la matriz en profundidad. El nivel freático en el sector se encuentra en el contacto de los depósitos de suelo con la roca basal. El contacto con la roca basal se encuentra a profundidades superiores a $100 \mathrm{~m}$. Se definieron dos estratos representativos de acuerdo a las características granulométricas que se describieron anteriormente. Los parámetros geotécnicos para cada material son los que se muestran en la Tabla 2:

Tabla 2: Parámetros geotécnicos de unidades de suelo

\begin{tabular}{|l|l|c|c|}
\hline \multicolumn{2}{|l|}{ Material } & $\begin{array}{c}\text { Grava limo } \\
\text { arenosa }\end{array}$ & Arena limosa \\
\hline \multicolumn{2}{|l|}{ Profundidad, $\mathrm{m}$} & $0-20$ & Mayor que 20 \\
\hline \multicolumn{2}{|l|}{ Peso específico sólidos, $G_{\mathrm{s}}$} & 2.65 & 2.65 \\
\hline \multirow{2}{*}{ Peso unitario, $\mathrm{t} / \mathrm{m}^{3}$} & Natural & 2.03 & 1.90 \\
\cline { 2 - 4 } & Seca & 2.00 & 1.87 \\
\hline \multirow{2}{*}{$\begin{array}{l}\text { Humedad } \\
\text { gravimétrica, \% }\end{array}$} & Natural & 1.5 & 1.5 \\
\cline { 2 - 4 } & Saturación & 12.3 & 15.7 \\
\hline \multirow{2}{*}{$\begin{array}{l}\text { Humedad } \\
\text { volumétrica, \% }\end{array}$} & Natural & 3.0 & 3.0 \\
\cline { 2 - 4 } & Saturación & 24.6 & 29.4 \\
\hline
\end{tabular}

\section{Metodología}

El modelo numérico requiere como parámetro de entrada las curvas características del suelo, con el fin de estimar el coeficiente de permeabilidad para una determinada succión. La metodología desarrollada en la determinación de las curvas se entrega en este apartado.

\section{Curvas características de contenido de agua}

A partir de los parámetros geotécnicos establecidos para cada estrato, se estimaron las curvas características de contenido de agua en el suelo SWCC (Soil Water Characteristic Curves), las que se validaron con ensayos de succión a muestras provenientes de sondajes. Estas curvas permiten representar la cantidad de agua albergada en los poros bajo una determinada succión. De acuerdo al método propuesto por Fredlund y Xing (1994), el contenido volumétrico de agua para un suelo parcialmente saturado en un rango de succión de 0 a $10^{6} \mathrm{kPa}$, se expresa como:

$$
\theta_{w}=C_{\psi} \frac{\theta_{s}}{\left(\ln \left(e+\left(\frac{\psi}{a}\right)^{n}\right)\right)^{m}}
$$

donde $\theta_{w}$ es el contenido volumétrico de agua $\left(\mathrm{m}^{3} / \mathrm{m}^{3}\right), C_{\psi}$ es una función de corrección, $\theta_{s}$ es el contenido volumétrico de agua de saturación $\left(\mathrm{m}^{3} / \mathrm{m}^{3}\right)$, e es el número de Euler igual a $2.71828, \psi$ es la presión de poros negativa (succión) en $\mathrm{kPa}$ y $a, n, m$ son parámetros de ajuste de curva.

Para predecir las curvas SWCC a partir de las curvas granulométricas se utilizó la función de pedo-transferencia de Fredlund et al. (2002) que se presenta en la ecuación (2).

$$
P_{p}(d)=\frac{1}{\left(\ln \left(e+\left(\frac{g_{a}}{d}\right)^{g_{n}}\right)\right)^{g_{m}}}\left(1-\left(\frac{\ln \left(1+\frac{d_{r}}{d}\right)}{\ln \left(1+\frac{d_{r}}{d_{m}}\right)}\right)^{7}\right)
$$

donde $P_{p}(d)$ es el porcentaje que pasa de una granulometría particular (\%), $d$ es el diámetro de partícula en $\mathrm{mm}, g_{a}$, $g_{n}, g_{m}$ son parámetros de ajuste correspondientes a la ruptura inicial, pendiente máxima y curvatura de la curva granulométrica respectivamente, $d_{m}$ es el diámetro de partícula mínimo en $\mathrm{mm}$ y $d_{r}$ es el diámetro de partícula residual en $\mathrm{mm}$.

Este modelo ajusta la curva granulométrica de un material, en primera instancia, a la ecuación (1) de Fredlund y Xing (1994). La curva de distribución del tamaño de partículas es luego analizada como una serie incremental del tamaño de partículas desde la más pequeña hasta la más grande para construir la curva SWCC en su totalidad. Para cada tamaño uniforme de partícula se obtiene una curva SWCC representativa del promedio de tamaño de partículas mediante una base de datos de 6000 suelos. Una vez que la curva de distribución granulométrica ha sido incrementalmente analizada en su totalidad, las curvas SWCC individuales son sobrepuestas para obtener la curva SWCC completa. Las curvas SWCC estimadas para las unidades de suelo Grava Limo Arenosa y Arena Limosa se presentan en las Figuras 2 y 3 respectivamente. Estas curvas posteriormente se introducen en los modelos numéricos de infiltración elaborados. 


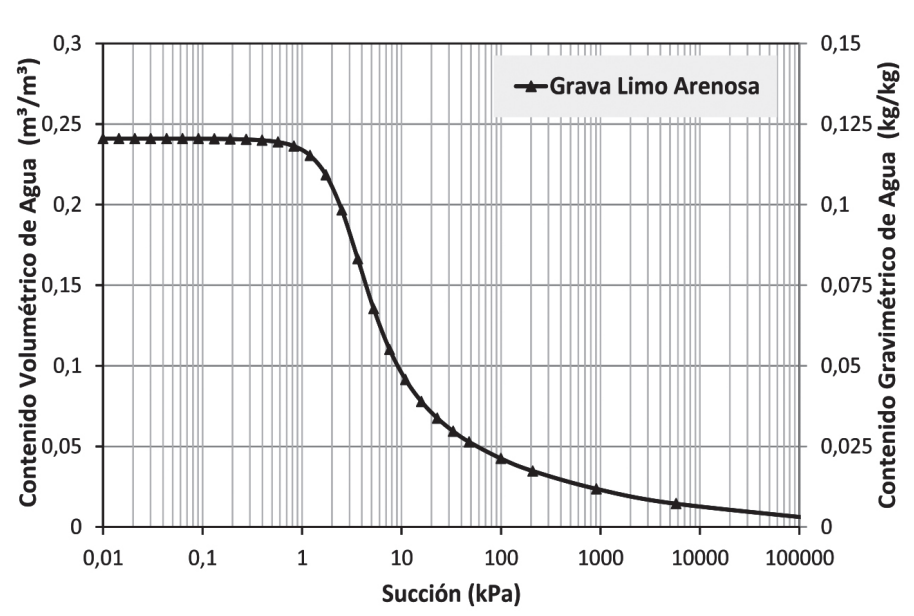

Figura 2: Curva SWCC, Grava Limo Arenosa

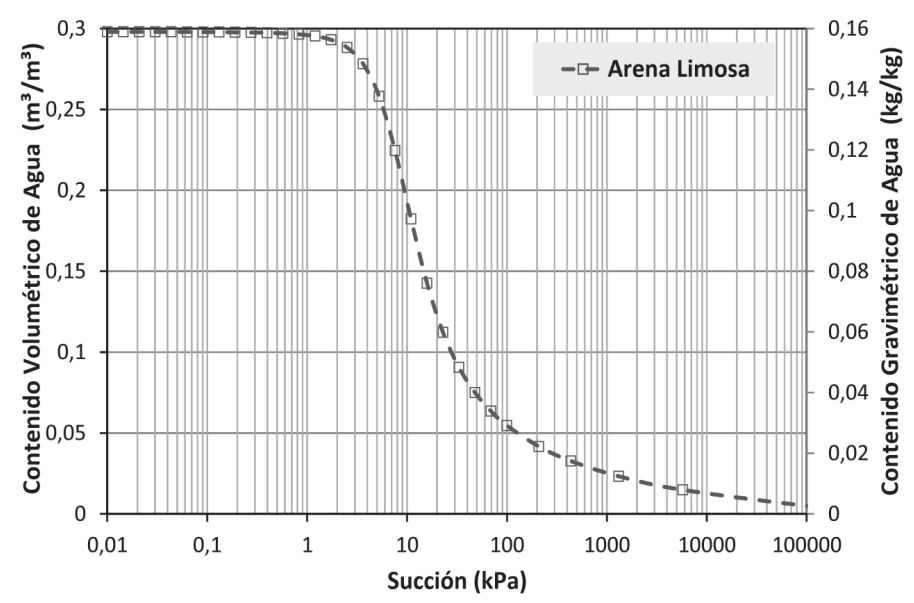

Figura 3: Curva SWCC, Arena Limosa

\section{Curvas de permeabilidad versus succión}

La estimación de los coeficientes de permeabilidad no saturada se efectuó por medio de la ecuación desarrollada por Fredlund et al. (1994) incluida en SEEP/W (Geostudio, 2010) a partir de las curvas SWCC, que relaciona el coeficiente de permeabilidad con el contenido volumétrico de agua:

$$
k_{w}=k_{s} \frac{\sum_{i=j}^{N} \frac{\theta\left(e^{y}\right)-\theta(\psi)}{e^{y_{i}}} \theta^{\prime}\left(e^{y_{i}}\right)}{\sum_{i=1}^{N} \frac{\theta\left(e^{y}\right)-\theta_{s}}{e^{y_{i}}} \theta^{\prime}\left(e^{y_{i}}\right)}
$$

donde $k_{w}$ es el coeficiente de permeabilidad para una succión específica en $\mathrm{m} / \mathrm{s}, k_{s}$ es el coeficiente de permeabilidad saturada en $\mathrm{m} / \mathrm{s}$, es una variable ficticia de la integración que representa el logaritmo de la presión de poros negativa, $i$ es el intervalo entre $j$ y $N$, siendo $j \mathrm{y}$ $N$ la succión mínima y máxima respectivamente, $\psi$ es la succión correspondiente a $j$; y $\theta^{\prime}$ es la primera derivada de la ecuación (1).

\section{Modelos de infiltración para estimar el co- eficiente de permeabilidad saturada}

A través de modelamiento numérico, se desarrollaron análisis de filtraciones que simularon los ensayos de permeabilidad de carga constante y carga variable ejecutados en los sondajes. Para esto, se utilizó el software de elementos finitos SEEP/W (Geostudio, 2010). Se analizaron modelos de carácter axialsimétrico en régimen transiente, con el fin de representar las condiciones de realización de cada ensayo. La condición inicial no saturada de ambos estratos de suelo definidas en este estudio, se consideró al evaluar cada material con un contenido inicial de humedad gravimétrica igual a $1.5 \%$, equivalente a succiones de $30 \mathrm{kPa}$ para el material Grava limo arenosa y $60 \mathrm{kPa}$ para Arena limosa, de acuerdo a las curvas SWCC (Figuras 2 y 3 respectivamente), propiedad hidráulica que varía en relación al frente de saturación.

Las condiciones de borde incorporadas en los modelos corresponden a una condición de flujo nulo $(\mathrm{Q}=0)$ en todo el eje de simetría, salvo en la longitud que representa la cámara del ensayo de permeabilidad para cada tramo, en la cual se introdujo una función que replica la carga hidrostática versus tiempo correspondiente al registro de lecturas propio del ensayo del tramo en cuestión. A continuación se llevó a cabo un proceso iterativo que consistió en asignar a cada tramo de ensayo en el sondaje, una determinada curva de conductividad hidráulica (permeabilidad) versus succión, estimada a partir de la curva SWCC representativa del material encontrado en dicho tramo, para posteriormente ir ajustando el valor de la permeabilidad saturada, hasta alcanzar en cada modelo un valor de caudal de infiltración igual o similar (variaciones en la segunda cifra significativa) al obtenido con las mediciones de los ensayos de permeabilidad en terreno. Para lograr lo expuesto en el párrafo anterior, en los modelos numéricos se efectuaron lecturas de caudales, calculando la variación de volumen de agua infiltrando al suelo en un determinado intervalo de tiempo. 


\section{Modelo de infiltración desarrollado para el Sondaje S-01}

A modo de ejemplificar la metodología propuesta en este estudio, se presenta el procedimiento empleado para la estimación del coeficiente de permeabilidad en los tramos de ensayo del sondaje denominado S-01. Este sondaje alcanzó una profundidad de $170 \mathrm{~m}$, los primeros $110 \mathrm{~m}$ de perforación en suelo y los restantes $60 \mathrm{~m}$, en roca. El nivel freático se observó a los $110 \mathrm{~m}$ de profundidad aproximadamente, justo en el contacto entre el depósito de suelo y la roca basal. En este sondaje se realizaron pruebas de permeabilidad en suelo, de carga constante y carga variable en ocho tramos, abarcando toda la longitud de sondaje ejecutado en suelo. Se simuló la ejecución de los ensayos de carga constante y de carga variable en cada uno de los tramos indicados.

De acuerdo a la caracterización geotécnica de los materiales involucrados, en el tramo T1 se consideró el uso de la curva SWCC del material Grava Limo Arenosa, mientras que en los tramos restantes (T2 a T8) se utilizó la curva SWCC del material definido como Arena Limosa. La posición del nivel freático se estableció a los $110 \mathrm{~m}$ de profundidad. En la Figura 4 se presenta de manera gráfica el modelo en elementos finitos desarrollado para el sondaje S-01, indicando los distintos tramos analizados, tamaño de elementos y condiciones de borde empleadas.
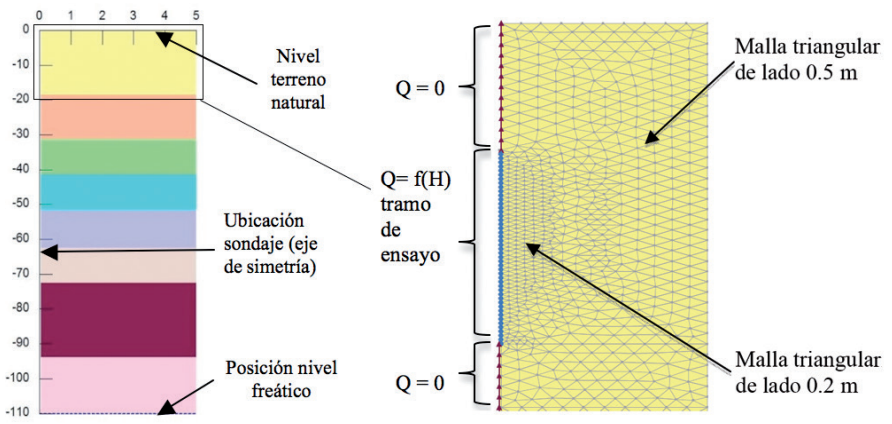

Figura 4: a) Modelo SEEP/W Sondaje S-01 y b) condiciones de borde y el diseño de la malla triangular de elementos finitos del tramo T1

Las profundidades de cada tramo, tiempos de simulación de cada tipo de ensayo, caudales medidos en terreno y la disposición del modelo numérico desarrollado para el Sondaje S-01, se presentan en la Tabla 3.
Tabla 3: Modelo de infiltración sondaje S-01, (dimensiones en m)

\begin{tabular}{|l|l|c|c|c|}
\hline \multirow{2}{*}{$\begin{array}{l}\text { Tramo } \\
\text { ensayo }\end{array}$} & \multirow{2}{*}{$\begin{array}{l}\text { Profundidad, } \\
\mathrm{m}\end{array}$} & \multicolumn{2}{|l|}{ Ensayo carga constante } & Tiempo \\
\cline { 3 - 4 } & Tiempo, s & Caudal, $\mathrm{l} / \mathrm{s}$ & $\begin{array}{l}\text { ensayo carga } \\
\text { variable, } \mathrm{s}\end{array}$ \\
\hline T1 & $6.1-15.4$ & 713 & 0.375 & 812 \\
\hline T2 & $21.5-31.5$ & 792 & 0.046 & 2871 \\
\hline T3 & $30.6-41.5$ & 743 & 0.007 & 3900 \\
\hline T4 & $40.6-51.5$ & 851 & 0.224 & 2871 \\
\hline T5 & $50.6-62.5$ & 792 & 0.111 & 2772 \\
\hline T6 & $62.5-72.5$ & 673 & 0.039 & 5148 \\
\hline T7 & $72.5-82.5$ & 693 & 0.116 & 3960 \\
\hline T8 & $93.9-108.9$ & 594 & 0.004 & 56430 \\
\hline
\end{tabular}

La Figura 5 muestra la variación del caudal de infiltración en función del tiempo obtenida para el tramo T2 del sondaje S-01, ajustado a un coeficiente de permeabilidad saturado $k_{s}=3.5 \cdot 10^{-8} \mathrm{~m} / \mathrm{s}$. Este análisis se desarrolló para cada tramo, calibrando el valor de $k_{s}$, y que al comparar con los registros de caudal medidos en cada prueba de permeabilidad en terreno se obtuviese la mejor aproximación posible.

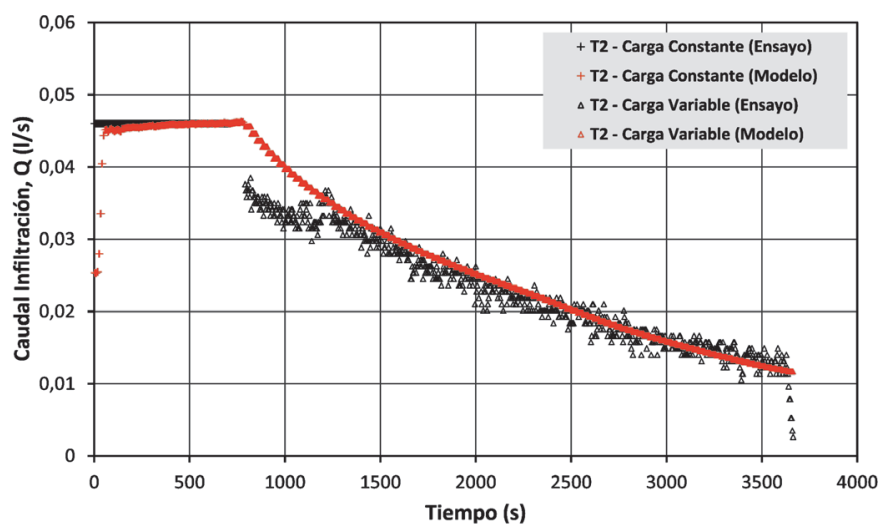

Figura 5: Variación del caudal de infiltración para el tramo T2, con $k_{s}=3.5 \cdot 10^{-8} \mathrm{~m} / \mathrm{s}$.

La Figura 6 muestra el bulbo de saturación que se genera una vez finalizado el proceso de simulación del ciclo de ensayos en el modelo numérico correspondiente al tramo T2. Se aprecia que el frente de saturación avanza una distancia menor que $50 \mathrm{~cm}$. 


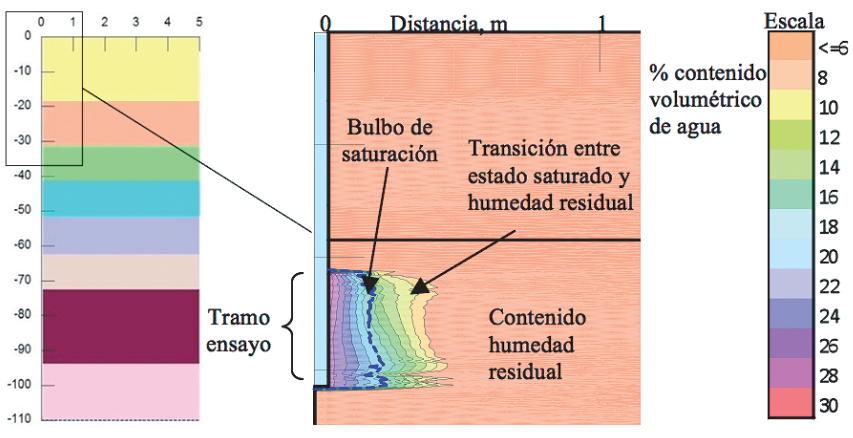

Figura 6: Bulbo de saturación modelo numérico tramo T2, sondaje S-01

El resumen de las curvas de permeabilidad obtenidas para cada tramo de análisis del sondaje S-01 se entrega en la Figura 7. El rango de permeabilidades saturadas varió entre $10^{-10}$ y $10^{-6} \mathrm{~m} / \mathrm{s}$ aproximadamente, alcanzando como máximo una succión de $5 \mathrm{kPa}$ para este rango.

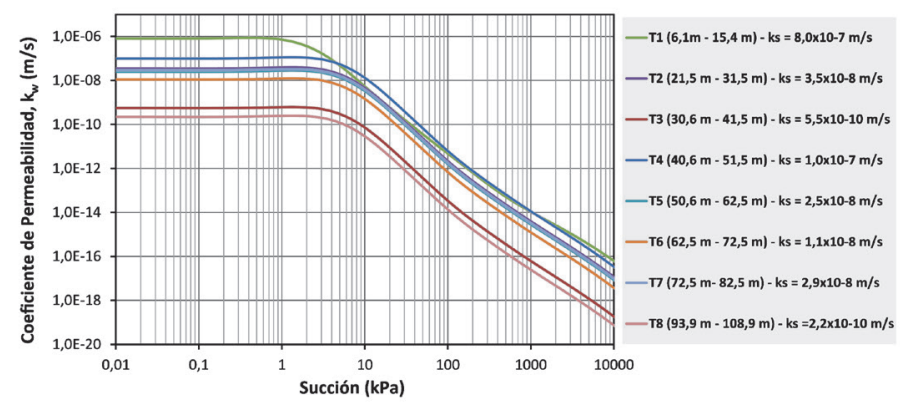

Figura 7: Curvas de permeabilidad versus succión, sondaje S-01

\section{Comparación de valores del coeficiente de permeabilidad saturada}

En la Figura 8 se comparan las permeabilidades obtenidas a partir del modelamiento numérico con aquellas obtenidas de manera simplificada a través de ensayos de Lefranc en régimen de carga constante. Este último supone una condición saturada del suelo, y se expresa de acuerdo a la ecuación (4).

$$
k_{s}=\frac{Q}{C h_{m}}
$$

Donde $k_{s}$ es el coeficiente de permeabilidad saturada en $\mathrm{m} / \mathrm{s}$, $Q$ es el caudal inyectado en el ensayo de carga constante en $\mathrm{m}^{3} / \mathrm{s}, h_{m}$ es la altura de agua dentro del sondaje, por encima del nivel estático previo en $\mathrm{m}$ y $C$ es un coeficiente de forma para un diámetro mucho menor al largo del sondaje, definido como:

$$
C=\frac{2 \pi L}{\ln (2 L / d)}
$$

en que $L$ es la longitud de la zona filtrante en m y $d$ es el diámetro de la zona filtrante en $\mathrm{m}$. Como se puede observar en la Figura 8, la permeabilidad determinada mediante los modelos numéricos es generalmente un orden de magnitud menor.

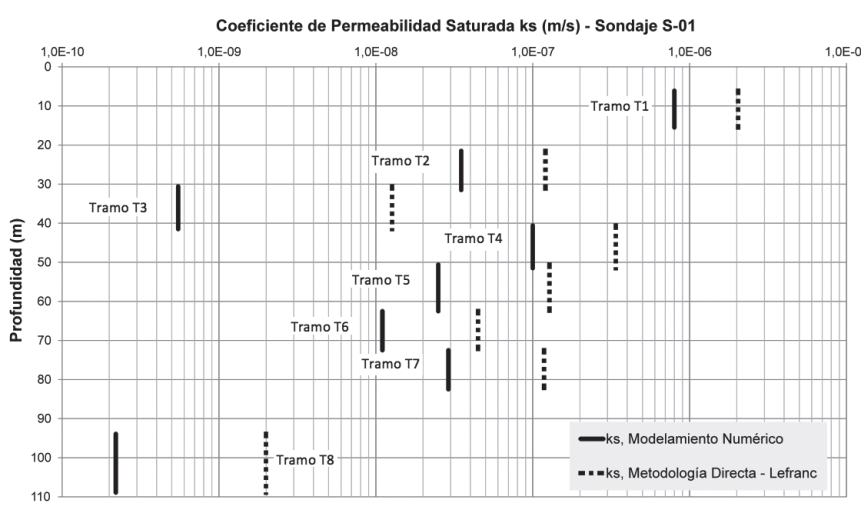

Figura 8: Valores del coeficiente de permeabilidad saturada obtenidos mediante modelamiento numérico y métodos de aplicación directa, Sondaje S-01.

\section{Conclusiones}

La aplicación de una solución analítica en la determinación de la permeabilidad saturada requiere de la toma de suposiciones que son sensibles a la variabilidad tanto vertical como lateral de los suelos. Esto es especialmente cierto en los métodos de ensayo tradicionalmente empleados en sondajes, lo que se traduce en un alto grado de incertidumbre en los resultados. Los modelos presentados en el presente artículo fueron utilizados en un proyecto desarrollado sobre depósitos aluviales no saturados profundos y realizados con equipos disponibles en la industria. Se considera que es un método con un alto grado de confiabilidad y de fácil aplicación para determinar la permeabilidad saturada en medios no saturados.

Los resultados presentados en este estudio indican que la metodología desarrollada mediante modelamiento numérico arroja en general valores de coeficiente de permeabilidad un orden de magnitud menor que los obtenidos de manera simplificada a través de ensayos de 
Lefranc en régimen de carga constante, el cual asume que el terreno se encuentra saturado. El posterior uso de las permeabilidades saturadas obtenidas del modelamiento numérico en una caracterización hidrogeológica con mayor confianza en los valores aplicados, puede traducirse por ejemplo en estimaciones de flujos subterráneos de menor magnitud y en diseños de obras relacionadas optimizados.

\section{Agradecimientos}

Los autores quieren expresar su gratitud a los ingenieros Claudia Vallejos y Terry Eldridge por su contribución en la elaboración de este artículo, así como a la compañía minera que permitió el desarrollo de estos trabajos.

\section{Referencias}

Chapuis, R.P. (1989). Shape factors for permeability tests in boreholes and piezometers. Groundwater 27(9): 649 - 654

Custodio, E. y Llamas, M.R. (1983). Hidrología Subterránea. Tomo II, 2a ed., editorial OMEGA S.A., Barcelona

Fredlund, D.G. and Xing, A. (1994). Equations for the soil-water characteristic curve. Canadian Geotechnical Journal 31(4), 521 - 532
Fredlund, M.D., Wilson, G.W. and Fredlund, D.G. (2002). Use of the grain-size distribution for the estimation of the soil-water characteristic curve. Canadian Geotechnical Journal 39(5), $1103-1117$

Fredlund, D.G., Xing, A. and Huang, S. (1994). Predicting the permeability function for unsaturated soils using the soil-water characteristic curve. Canadian Geotechnical Journal 31(4): 533-546

Geostudio (2010). Seepage Modeling with SEEP/W 2007. An Engineering Methodology, Geo-Slope International Ltd. $4^{\text {th }}$ ed; Calgary

Hvorslev, M.J. (1951). Time lag and soil permeability in groundwater observations. US Army Waterways Experiment Station, Corps of Engineers, Vicksburg, Mississippi. Bulletin №36

Philip, J.R. (1985). Approximate analysis of the borehole permeameter in unsaturated soil. Water Resources Research 21(5), $1025-1033$ 